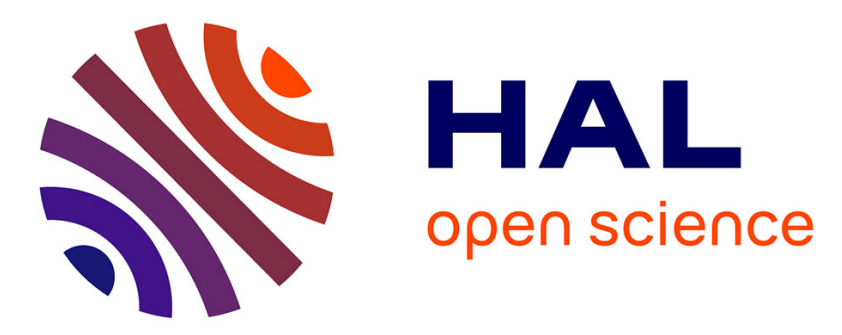

\title{
The chord length distribution of a two-sphere aggregate
} Frédéric Gruy, Sandra Jacquier

\section{To cite this version:}

Frédéric Gruy, Sandra Jacquier. The chord length distribution of a two-sphere aggregate. Computational Materials Science, 2021, 44 (2), pp.218-223. 10.1016/j.commatsci.2008.03.026 . emse03434903

\section{HAL Id: emse-03434903 \\ https://hal-emse.ccsd.cnrs.fr/emse-03434903}

Submitted on 18 Nov 2021

HAL is a multi-disciplinary open access archive for the deposit and dissemination of scientific research documents, whether they are published or not. The documents may come from teaching and research institutions in France or abroad, or from public or private research centers.
L'archive ouverte pluridisciplinaire HAL, est destinée au dépôt et à la diffusion de documents scientifiques de niveau recherche, publiés ou non, émanant des établissements d'enseignement et de recherche français ou étrangers, des laboratoires publics ou privés. 


\title{
The chord length distribution of a two-sphere aggregate
}

\author{
Frédéric Gruy * and Sandra Jacquier \\ École Nationale Supérieure des Mines de Saint-Étienne, Centre SPIN, \\ 158 Cours Fauriel 42023 St. Étienne, France
}

\begin{abstract}
Mono-disperse particle suspensions are widely used in the field of particle technology. However, particles may aggregate. As a consequence, the presence of aggregates changes the properties of the suspension. The monitoring of the ageing suspension can be performed by using light extinction or turbidity measurements (TM). Intensity attenuation is depending on the particle concentration and on their scattering cross section. The latter is related to the chord length distribution (CLD) of particles. At the beginning of the aggregation process, only spherical particles and two-sphere aggregates are present in suspension. Thus, interpretation of turbidity signal needs the knowledge of the chord length distribution of spheres and twosphere aggregates. This paper presents a calculation of CLD for a two-sphere aggregate available for explaining turbidity measurements (CLD-TM). The corresponding expression is compared to the CLD obtained by simulation.
\end{abstract}

Keywords: Chord Length Distribution (CLD), Doublet, Aggregation, Turbidity, Anomalous Diffraction

\section{Introduction}

Many manufacturers use solid micro-particles in suspension for various applications: ceramics, paintings, pharmaceutics, cosmetics, food, chemicals. These suspended particles may aggregate ([1-2]) under the influence of a mechanical constraint such as stirring or a chemical constraint such as $\mathrm{pH}$ variation. An aggregate is a set of particles, known as primary particles connected between them. Aggregates modify the initial suspension properties such as filterability, or bring about new final product properties.

The aggregation process consists in several stages [3]:

- the collision stage : collision between two primary particles gives rise to a two-sphere set or a doublet,

- the formation stage: formation of larger aggregate,

- the fragmentation stage: fragmentation of the larger aggregates which leads to a decrease of the aggregation rate,

- the equilibrium stage: appearance of a dynamic balance between the aggregation and fragmentation processes. This leads to a maximum size for the aggregates.

The objects, made during aggregation, are constituted by a variable primary particles number. The smallest aggregate is the doublet. Some knowledge can be obtained from the beginning of aggregation, i.e doublet formation.

*Corresponding author. Tel :+33(0)4 77420057 ; fax :+33(0)4 77499694

E-mail address : fgruy@emse.fr (F. Gruy),jacquier_sj @yahoo.fr 
In order to monitor aggregation in situ, the interaction between light and particles is used. There are already commercial devices using this non-invasive method. The two main in situ methods for micro-particle sizing are Turbidity Measurement (TM) and Focused-Beam Reflectance Measurement (FBRM). Depending on the optical contrast between particles and suspending medium, measurements can be directly related to the chord length distribution (CLD) [4-8].

CLDs are involved in several physical devices in order to characterize suspensions of randomly orientated identical particles. These characterization methods are based on the scattering between the particle and an incident electromagnetic wave. The scattered wave is depending on the particle morphology and on the ratio $m$ between the refractive indices of particle material and suspending medium. One way to quantify morphology is to determine the CLD. However, CLD can be defined by different ways. This is illustrated by three examples.

- Small-Angle Scattering (SAS) measurements lead to the electromagnetic wave intensity $I$ as a function of the scattering wave vector $(k=4 \pi / \lambda \sin (\vartheta / 2)) . \lambda$ and $\vartheta$ are respectively the radiation wavelength and the scattering angle. The intensity is related to the CLD by the relation [9]:

$I(k)=\frac{\int_{0}^{L} P(r) \sin (k r) /(k r) 4 \pi r^{2} d r}{\int_{0}^{L} P(r) 4 \pi r^{2} d r}$

with $\quad D(r)=\frac{4 V}{S} \frac{d^{2} P}{d r^{2}}$

$P(r)$ is the probability that a point located at the distance $r$ from a point located inside the object is inside the object. $S$ and $V$ are respectively the surface area and the volume of the object. The relation Eq. (1) is applicable for convex object. The chord length distribution $D(r)$ is obtained if the geometric figure is exposed to an isotropic uniform flow of infinite straight lines. SAS measurements are suitable for nano-particles interacting with $\mathrm{X}$ rays. Moreover the method can be extended to optically soft micro-particles interacting with light [10]. The corresponding method is called Static Light Scattering (SLS). So, Gille calculated $D(r)$ for various shapes: hemisphere [11], two-cylinders set [12], triangular rod [13], hollow cylinder [14], cylinders [15].

- Focused Beam Reflectance Measurements (FBRM) are among the most widely techniques for particle sizing [7]. It uses a focused beam of laser light that scans across particles passing in front of the probe window to measure a chord length distribution. The interpretation of the signal is only based on the reflected light. This method is suitable for particle size higher than $5 \mu \mathrm{m} . D(r)$ results if each projected area of the geometric figure is exposed to a 2D isotropic uniform flow of infinite straight lines.

- Turbidimetry, i.e extinction measurement, is an optical method to measure the light scattering or extinction cross section of particles. In the case of large particles $(>1 \mu \mathrm{m})$ and very small optical contrast $(|m-1|<<1)$, extinction or scattering can be explained in the framework of anomalous diffraction approximation [10]: the refracted rays propagate almost without a change of direction and interfere with the diffracted component. An important optically soft material is silica. Anomalous diffraction approximation was 
applied to a sphere and an infinitely long circular cylinder, a prism column, a hexagonal crystal of ice, ellipsoids and a finite cylinder [16].

In this case, the scattering cross section $C$ (non absorbing particle) obeys:

$$
C=2 \iint_{[S p]}\left[1-\cos \left(k_{0} l(m-1)\right)\right] d S_{p}
$$

Integration is performed over the particle projected area $S_{p}$ on a plane perpendicular to propagation direction. $l$ is the computed path of light through the particle, which is a function of the projection coordinates $\mathrm{x}$ and $\mathrm{y} . k_{0}$ is the wave vector in the suspending medium.

Average $\mathrm{C}(<\mathrm{C}\rangle)$ is obtained while rotating the particle on itself (or by changing the projection plane) and then by carrying out the average over all the discrete angle values.

$<C>=2\left\langle\iint_{[S p]}\left[1-\cos \left(k_{0} l(m-1)\right)\right] d S_{p}\right\rangle_{\theta}$

It can be shown that a good approximation for Eq. (3) is [5]:

$<C>=2<S_{p}>\int_{0}^{l_{\max }}\left[1-\cos \left(k_{0} l(m-1)\right)\right] D(l) d l$

chord length.

$\left\langle S_{p}\right\rangle$ is the projected area averaged over all orientations. $l_{\max }$ is the maximum

The goal of this paper is the exact calculation of the chord length distribution for a doublet of identical and spherical particles (the smallest possible aggregate) suitable for turbidity measurements.

It may be underlined that the definition of the CLD for a given object changes depending on the physical principles of measurement: the way to obtain the chord length distribution corresponding to TM (CLD-TM) and FBRM (CLD-FBRM) is illustrated on Fig. 1. The main difference is that:

- in the first one, the projected area is used to define the coordinates of the chord which goes across the doublet (Fig. 1a).

- $\quad$ in the second one, the chord goes through the doublet projected area (Fig. 1b).

Throughout the paper, the chord length distribution (density) is written $D(l) . D(l) d l$ is the number of chords within the $l$-range $[l, l+d l] . D(l)$ is normalized, i.e $\int_{0}^{l_{\max }} D(l) d l=1$.

The section two develops a methodology in order to calculate the CLD-TM of a two-sphere aggregate. It is followed by a discussion and a conclusion in the section three.

\section{Calculation of the chord length distribution}

\subsection{Definition of the different zones}

The doublet projection on a plane is considered. $\theta$ is the angle between the line binding the centres of the two spheres (radius $r$ ) and the projection plane (coordinates $x, y$ ). The centre 
of one of the two spheres is the origin $\mathrm{O}$ of the coordinates system (Fig. 2). The length of the chord $(l)$ which crosses one sphere or the two spheres obeys the equations (Eqs. 5a-c):

$$
\begin{aligned}
& l=2\left(r^{2}-d^{2}\right)^{1 / 2} \quad \text { if } \quad d<r, d^{\prime}>r \\
& l=2\left(r^{2}-d^{\prime 2}\right)^{1 / 2} \quad \text { if } \quad d>r, d^{\prime}<r \\
& l=2\left(r^{2}-d^{2}\right)^{1 / 2}+2\left(r^{2}-d^{\prime 2}\right)^{1 / 2} \quad \text { if } \quad d<r, d^{\prime}<r
\end{aligned}
$$

with

$d=\left(x^{2}+y^{2}\right)^{1 / 2} \quad$ and $\quad d^{\prime}=\left((x-2 r \cos (\theta))^{2}+y^{2}\right)^{1 / 2}$

The locus of the points $(x, y)$ with constant $l$ may be a circle (Eqs. 5a-b) or an ellipse (Eq. 5c):

$$
\frac{(x-r \cos (\theta))^{2}}{(r a)^{2}}+\frac{y^{2}}{(r b)^{2}}=1
$$

with

$$
a=\left[\frac{\sin ^{2}(\theta)-T}{1+T^{-1} \cos ^{2}(\theta)}\right]^{1 / 2}, \quad b=\left[\sin ^{2}(\theta)-T\right]^{1 / 2} \text { and } \quad T=l^{2} /\left(16 r^{2}\right)
$$

This ellipse exists if $\sin (\theta)>\sin \left(\theta_{0}\right)=l /(4 r)$ for a given $l$ value.

Let us denote $x_{1}=T / \cos (\theta)$. The intersection of the ellipse with the sphere of centre $\mathrm{O}$ exists if $x_{1}+\cos (\theta)<1$ (Fig. 3a). The coordinates of the intersecting point are: $x_{2}=r\left(x_{1}+\cos (\theta)\right)$ and $y_{2}=r\left(1-\left(x_{1}+\cos (\theta)\right)^{2}\right)^{1 / 2}$.

If $x_{1}+\cos (\theta)>1$, there is no intersection (Fig. 3b).

One defines the angles $\theta_{+}, \theta_{-}$as:

$\cos \left(\theta_{-}\right)=\left(1-\left(1-l^{2} /\left(4 r^{2}\right)\right)^{1 / 2}\right) / 2 \quad$ and $\quad \cos \left(\theta_{+}\right)=\left(1+\left(1-l^{2} /\left(4 r^{2}\right)\right)^{1 / 2}\right) / 2$

which are the roots of $\cos (\theta)+x_{1}=1$ ( if $l<2 r$ ).

It is easily checked: $0<\theta_{0}<\theta_{+}<\theta_{-}<\pi / 2$.

Firstly, the contributions of the different zones of the projected area to the CLD will be calculated $(\$ 2.2,2.3$ and 2.4). Then, the chord length distribution for the doublet will be deduced $(\$ 2.5)$.

2.2. Sum chords: calculation of the CLD for chords which cross both spheres one after the others (Fig. 4a)

As the number of chords perpendicularly crossing a projection plane and the particle with a chord length within the range $[l, l+d l]$ is proportional to the corresponding projected area element, the relation between the projected area $S$ and the chord length distribution is:

$$
D(l)=N \frac{d S}{d l}
$$

$N$ is a normalization factor which will be specified later.

Depending on the calculation, the area $S$ is the part of the projected area connected with a chord length smaller or higher than a given $l$ value. 
The fraction of the projected area, such as the chord length is smaller than $l$, obeys the equations:

$$
\begin{aligned}
& \quad-\text { if } x_{1}+\cos (\theta)<1 \\
& S_{1}=4 r^{2} \int_{0}^{x_{1}}\left[\left(1-(x+\cos (\theta))^{2}\right)^{1 / 2}-b\left(1-x^{2} / a^{2}\right)^{1 / 2}\right] d x \\
& \quad-\text { if } x_{1}+\cos (\theta)>1 \\
& S_{2}=2 r^{2}(\theta-\sin (2 \theta))-\pi r^{2} T^{1 / 2}\left[\left(T+\cos ^{2}(\theta)\right)^{-1 / 2}-\left(T+\cos ^{2}(\theta)\right)^{1 / 2}\right]
\end{aligned}
$$

$S_{1}$ and $S_{2}$ are shown on the Fig. $4 \mathrm{~b}-4 \mathrm{c}$.

The average CLD over all the orientations will be such as:

- if $l>2 r \quad D_{\alpha}(l)=N \int_{\theta_{0}}^{\pi / 2} d S_{2} / d l \cos (\theta) d \theta$

-if $l<2 r$

$$
D_{\alpha}(l)=N \int_{\theta_{+}}^{\theta_{\bar{C}}} d S_{1} / d l \cos (\theta) d \theta+N \int_{\theta_{0}}^{\theta_{+}} d S_{2} / d l \cos (\theta) d \theta+N \int_{\theta_{-}}^{\pi / 2} d S_{2} / d l \cos (\theta) d \theta
$$

With

$$
\begin{aligned}
& d S_{1} / d l=2 r^{2} x_{1} / l \frac{\cos (\theta)}{\left(x_{1}+\cos (\theta)\right)} \\
& {\left[\left(1-\left(x_{1}+\cos (\theta)\right)^{2}\right)^{1 / 2}-\left[\frac{1-\left(\cos (\theta)+2 x_{1}\right)\left(x_{1}+\cos (\theta)\right)}{x_{1}^{1 / 2}\left(x_{1}+\cos (\theta)\right)^{1 / 2}}\right] \arcsin \left(\frac{x_{1}^{1 / 2}\left(x_{1}+\cos (\theta)\right)^{1 / 2}}{\left(1-\cos (\theta)\left(x_{1}+\cos (\theta)\right)\right)^{1 / 2}}\right)\right]}
\end{aligned}
$$

and

$$
d S_{2} / d l=\frac{\pi}{l} r^{2} x_{1}^{1 / 2}\left(x_{1}+\cos (\theta)\right)^{-3 / 2} \cos (\theta)\left[2 x_{1}^{2}+3 x_{1} \cos (\theta)-1+\cos ^{2}(\theta)\right]
$$

\subsection{Calculation of the CLD for chords which cross a lens (Fig. 5)}

The previously calculated chord length distribution (crossing 2 spheres) corresponds to the intersection of the projected areas for the 2 spheres. The sphere chord length distributions corresponding to the shared part of their projected area must be subtracted from $D_{\alpha}(l)$.

Let us consider the sphere of centre $\mathrm{O}$ and its projection. The area element $d S$ belonging to the two projected areas and having the chord length in the range $[l, l+d l]$, is:

$$
\begin{array}{rlll}
\text { - if } \cos (\theta)>1 / 2 d S_{\varphi}=2 r_{1} \varphi d r_{1} & \text { with } & r_{1} \in[r(2 \cos (\theta)-1), r] \\
\text { - if } \cos (\theta)<1 / 2 d S_{\pi}=2 r_{1} \pi d r_{1} & \text { with } & r_{1} \in[0, r(1-2 \cos (\theta))] \\
d S_{\varphi}=2 r_{1} \varphi d r_{1} & \text { with } & r_{1} \in[r(1-2 \cos (\theta)), r]
\end{array}
$$

The angle $\varphi$ and the radius $r_{1}$ are depending on $l$ : 


$$
\begin{aligned}
& r_{1}=r\left(1-l^{2} /\left(4 r^{2}\right)\right)^{1 / 2} \\
& \varphi=\arccos \frac{\cos (\theta)-x_{1}}{\left(1-4 x_{1} \cos (\theta)\right)^{1 / 2}}
\end{aligned}
$$

The average (for the 2 spheres) over all the orientations is written:

$D_{\beta}(l)=-2 N \int_{\theta_{+}}^{\theta_{\overline{-}}} d S_{\varphi} / d l \cos (\theta) d \theta-2 N \int_{\theta_{-}}^{\pi / 2} d S_{\pi} / d l \cos (\theta) d \theta$

with $\quad d S_{\varphi} / d l=-8 x_{1} r^{2} / l \cos (\theta) \arccos \frac{\cos (\theta)-x_{1}}{\left(1-4 x_{1} \cos (\theta)\right)^{1 / 2}}$

and $\quad d S_{\pi} / d l=-8 \pi r^{2} x_{1} / l \cos (\theta)$

2.4. Calculation of the CLD for chords which cross a single sphere (Fig. 6)

The chord length distribution of the 2 spheres considered alone is obtained while taking $\varphi=\pi$ :

$D_{\gamma}(l)=2 N \pi(l / 2)$

\subsection{Calculation of the CLD for the doublet}

Thus, the chord length distribution of the doublet is:

$D(l)=D_{\gamma}(l)+D_{\alpha}(l)-D_{\beta}(l)$

The normalization factor $N$ is the inverse of the doublet projected area averaged over all the orientations:

$$
N^{-1}=\left\langle S_{p}\right\rangle_{\theta}=\int_{0}^{\pi / 2} S_{p} \cos (\theta) d \theta
$$

With

$$
S_{p}=2 r^{2}(\pi-\theta+\cos (\theta) \sin (\theta))
$$

Then

$$
N^{-1}=r^{2}(\pi+8 / 3)
$$

Note: The expression of $D(l)$ for the $l$-range $[2 r ; 4 r]$ can be deduced from Eqs. $(8 \mathrm{a}, 9 \mathrm{~b})$ :

$$
D_{\alpha}(l)=N r^{2} \frac{\pi}{l} T^{1 / 2}\left[T^{1 / 2} \frac{1-T}{1+T}+\frac{3 T-1}{2}\left(\arcsin \left((1+T)^{-1 / 2}\right)-\arcsin \left(T^{1 / 2}(1+T)^{-1 / 2}\right)\right)\right]
$$

The expression Eq. (15) was used in [5]. 


\section{Discussion and conclusion}

Fig. 7 presents the chord length distribution for a doublet of identical spheres by using the analytical method (Eq.(13)). The CLD of a single sphere is drawn on the same figure. Fig. 8 emphasizes the difference between a single sphere and a doublet of spheres. The comparison between analytical and simulated results can be seen on the same figure. Simulation is obtained by a previously published procedure [5]. Simulated and calculated CLD are in good agreement.

Two chord length ranges can be observed:

- $[0,2 r]$ : the CLD of the doublet is similar to the one of a sphere. The deviations are within 13 percent. This range contains the main contribution to the CLD.

- $[2 r, 4 r]$ : the corresponding contribution to the CLD is small. The maximum value of the chord length density is 0.0436 .

It can be also seen from Fig. 8 that the deviation of the doublet CLD from the sphere CLD is not symmetrical around $l=2 r$.

Operating with the analytic representation of the CLD, Eq. (4) can be applied for expressing the change of turbidity during the initial stage of aggregation. This will lead to the initial aggregation rate. 


\section{References}

[1] C. Tontrup, F. Gruy, and M. Cournil, Journal of colloid and interface science, 2000. 229: p. 511-525.

[2] F. Gruy, Journal of colloid and interface science, 2001. 237: p. 28-39.

[3] G.D. Boer, G. Hoedemakers, and D. Thoenes, Chemical Engineering Research and Design, 1989. 67: p. 301-315.

[4] S. Jacquier and F. Gruy, Journal of Quantitative Spectroscopy and Radiative Transfer, 2007. 106(1-3): p. 133-144.

[5] S. Jacquier and F. Gruy, Journal of Quantitative Spectroscopy and Radiative Transfer, 2008. 109:p. 789-810.

[6] E.J.W. Wynn, Powder Technology, 2003. 133: p. 125-133.

[7] M. Li and D. Wilkinson, Chemical Engineering Science, 2005. 60: p. 3251-3265.

[8] M-N Pons, K. Milferstedt, and E. Morgenroth, Chemical Engineering Science, 2006. 61: p. 3962-3973.

[9] A. Guinier and G. Fournet, Small-Angle scattering of X-rays, Wiley, New York, 1955

[10] H.C. Van de Hulst, Light scattering by small particles, John Wiley, NewYork, 1957.

[11] W. Gille, Computational Materials Science, 1999, 15 : p. 449-454

[12] W. Gille, Computational Materials Science, 2001, $20:$ p. 181-195

[13] W. Gille, Computational Materials Science, 2001, 22 : p. 151-154

[14] W. Gille, Mathematical and Computer Modelling, 2001, 34 : p. 423-431

[15] W. Gille, Powder Technology, 2002, 123 : p. 292-298

[16] A.A. Kokhanovsky, Optics of light scattering media, Springer, Berlin, 2001 


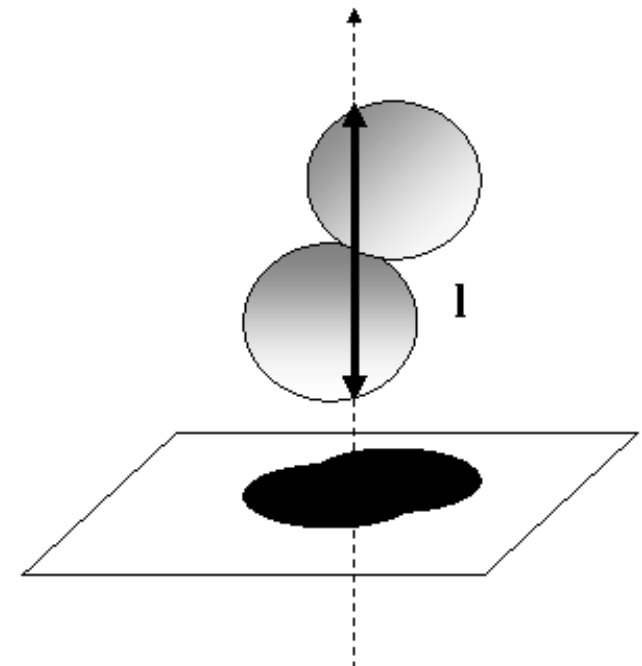

a)

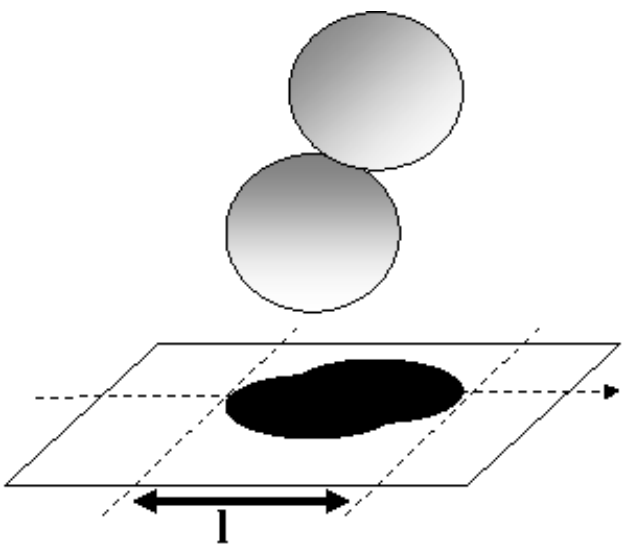

b)

Fig. 1. : Definition of a chord: CLD-TM a) and CLD-FBRM b)

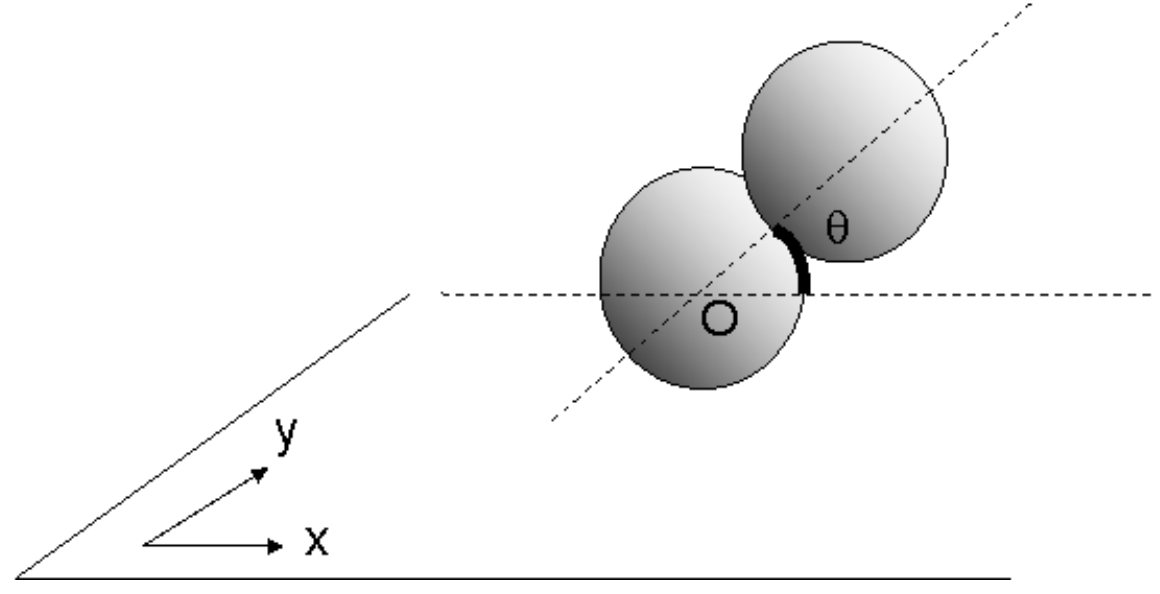

Fig.2: 3D drawing of the two-sphere aggregate

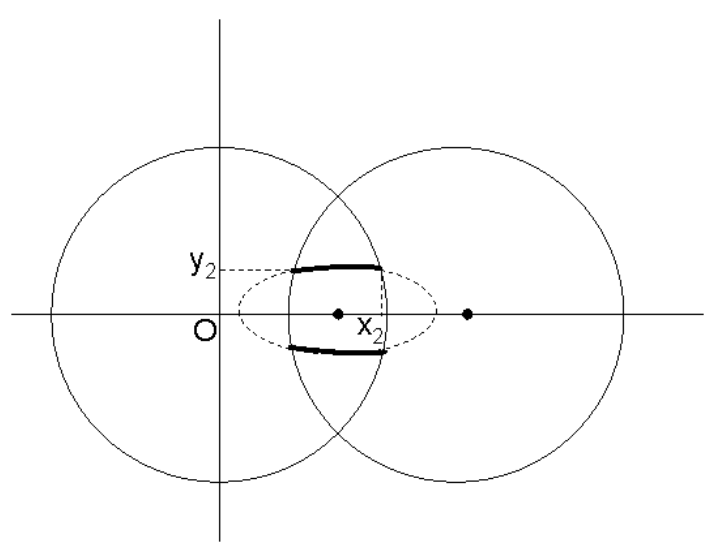

Fig.3a: curve (ellipse) corresponding to a constant $l$ value (projection plane) Case $x_{1}+\cos (\theta)<1$ 


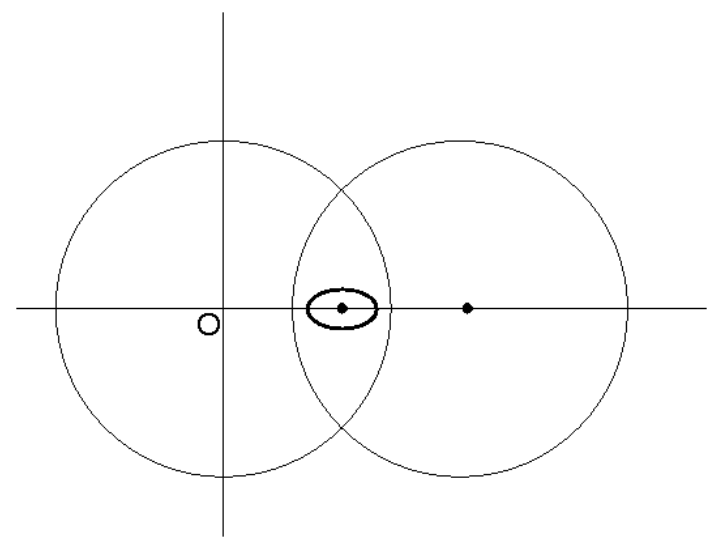

Fig.3b: curve (ellipse) corresponding to constant $l$ value (projection plane)

Case $x_{1}+\cos (\theta)>1$

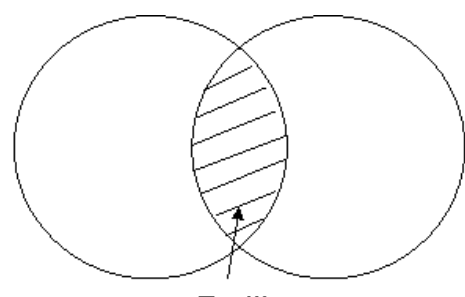

$\mathrm{D}_{\alpha}(\mathrm{I})$

Fig. 4a: $D_{\alpha}(l)$ represents the CLD from the dashed area (projection plane).

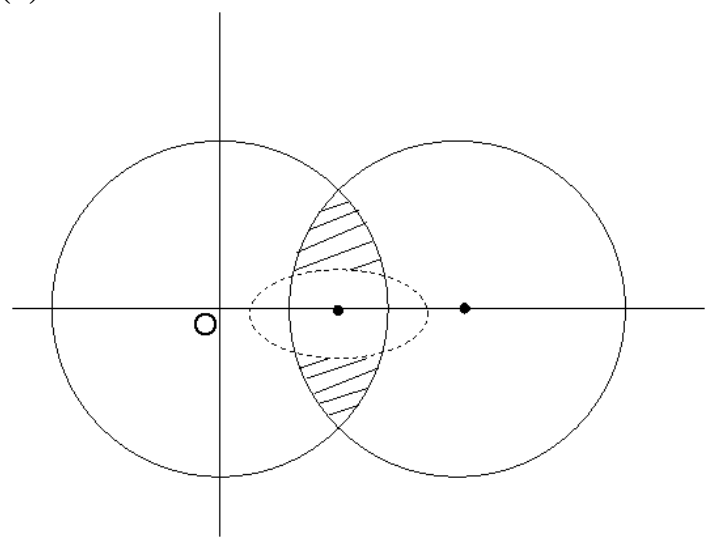

Fig. 4 b: the dashed area is denoted $S_{1}$ (projection plane)

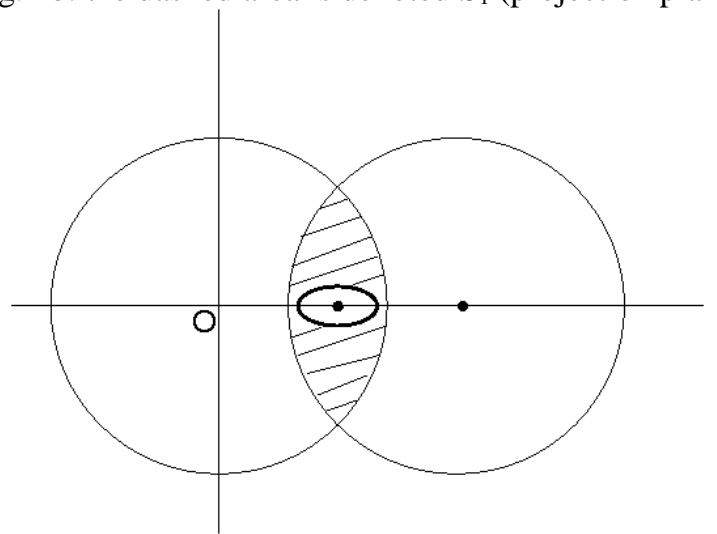

Fig. 4c: the dashed area is denoted $S_{2}$ (projection plane) 


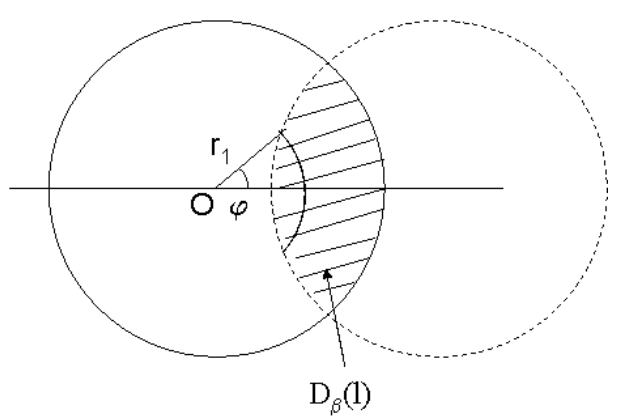

Fig.5: $D_{\beta}(l)$ represents the CLD from the dashed area (projection plane).

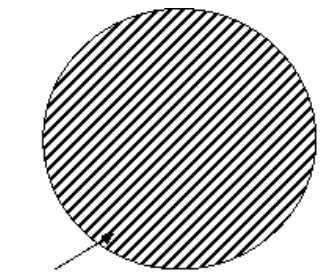

$D_{\gamma}(I)$

Fig. 6: $D_{\gamma}(l)$ represents the CLD from the dashed area (projection plane). 


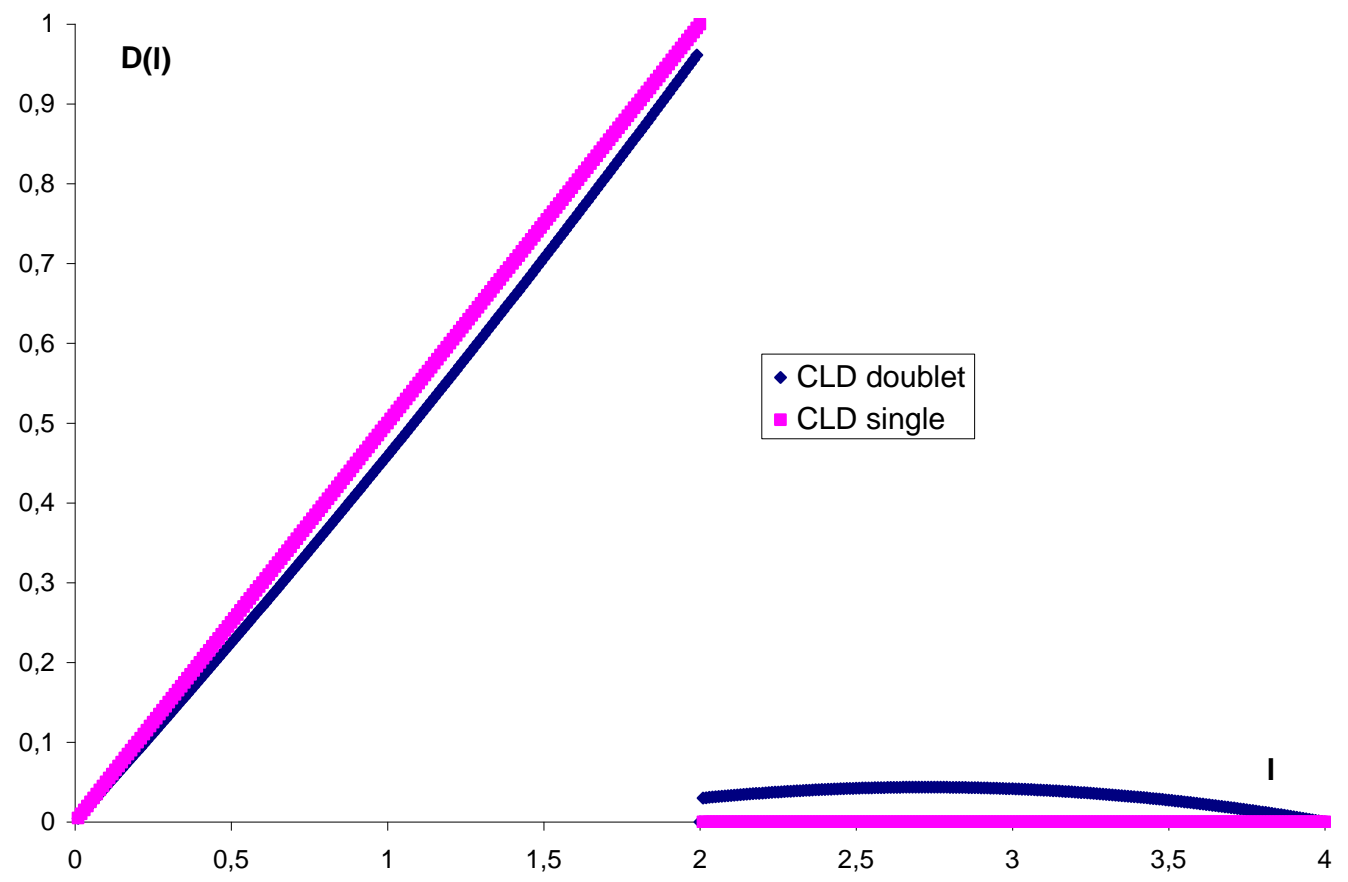

Fig. 7: CLD for a single sphere and a doublet of spheres $(\mathrm{r}=1)$

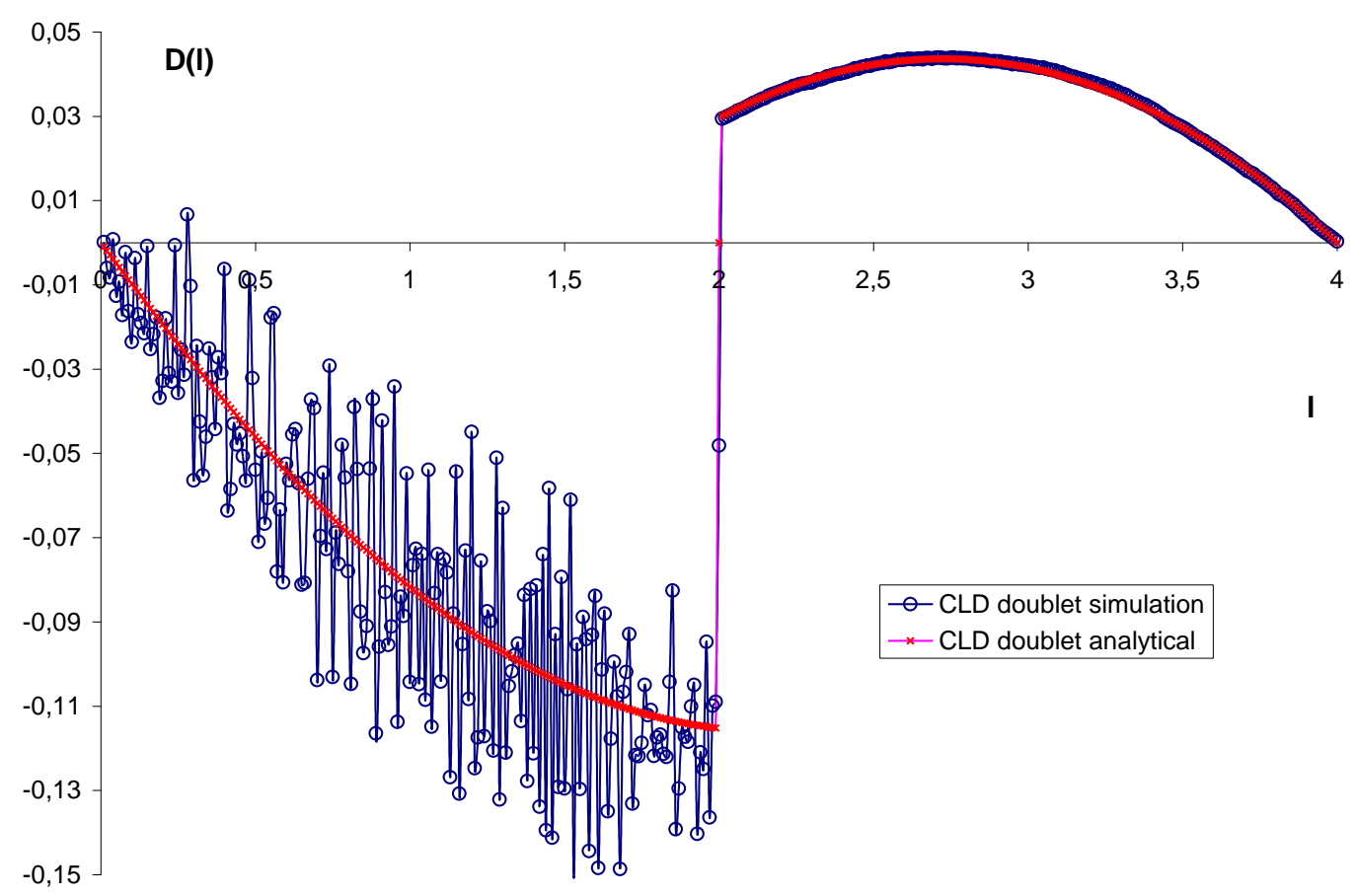

Fig. 8: difference between CLD of a doublet of spheres and a single sphere $(\mathrm{r}=1)$ 